\title{
EFFECT OF GRIPPING IN A TRIGGER POSTURE ON APPARENT MASS OF THE HAND-ARM SYSTEM
}

\author{
Neil J Mansfield*1, Nobuyuki Shibata ${ }^{2}$, Kazuma Ishimatsu ${ }^{2}$, Setsuo Maeda ${ }^{2}$ \\ ${ }^{1}$ Department of Ergonomics \\ Loughborough University \\ Loughborough \\ LE11 3TU \\ UK \\ 2 Japan National Institute of \\ Occupational Safety and Health \\ Nagao 6-21-1, Tama-ku \\ Kawasaki, 214-8585 \\ JAPAN
}

\section{Introduction}

Exposure to hand-arm vibration can induce hand-arm vibration syndrome (HAVS), one aspect of which is vibration white finger (VWF). The risk of developing HAVS is related to exposure duration, and the frequency and magnitude of the vibration. As the hand-arm system is not rigid, the extent to which forces are transmitted to the hand is a function of frequency and can be measured using the apparent mass, where a high apparent mass corresponds to higher forces being transmitted when compared to frequencies with a lower apparent mass. The apparent mass is affected by the direction of the vibration and the gripping conditions ${ }^{1,2}$.

When workers are exposed to hand-arm vibration from power tools, they often need to control a trigger. On most construction tools triggers are operated with the first and second finger whilst the thumb, third and fourth finger provide the primary grip for the tool in that hand. This paper reports an experiment to compare the biomechanical response of the hand-arm system when gripping with a 'full' grip and with a 'trigger' grip.

\section{Methods}

8 male subjects were exposed to triaxial random $(10-1000 \mathrm{~Hz})$ vibration. The vibration had a magnitude of $18 \mathrm{~m} / \mathrm{s}^{2}$ r.m.s. in each direction and was presented through a 40 $\mathrm{mm}$ handle containing accelerometers and force cells. Subjects gripped the handle at $30 \mathrm{~N}$ and pushed at $50 \mathrm{~N}$. Tests were conducted with

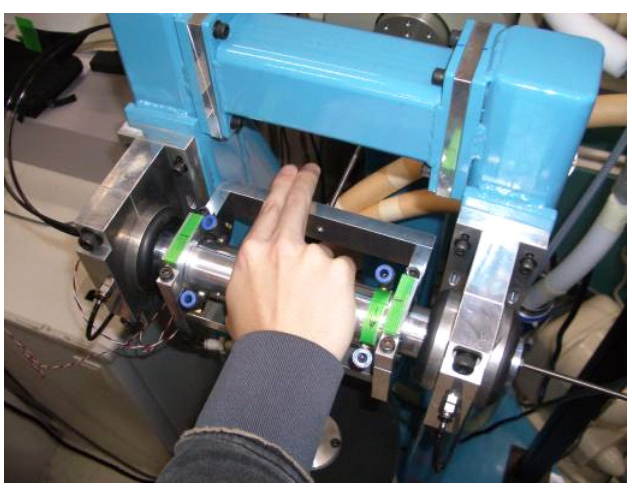

Figure 1. 'Trigger' grip posture. a straight (elbow $180^{\circ}$ ) and bent arm (elbow $90^{\circ}$ ). In the 'full' grip condition all fingers were used; in the 'trigger' grip there was no contact of the first two fingers with the handle (Figure 1). Apparent mass was measured separately for finger and palm sides of the hand.

\section{Results and Discussion}

Results for the 'full' grip showed similar trends to those in the literature ${ }^{1,2}$ (Figure 2 ). In the $x$ - and $y$-axes, the apparent mass in the 'trigger' grip posture was increased for the palm side at low frequencies. On the finger side apparent mass reduced, as expected, 

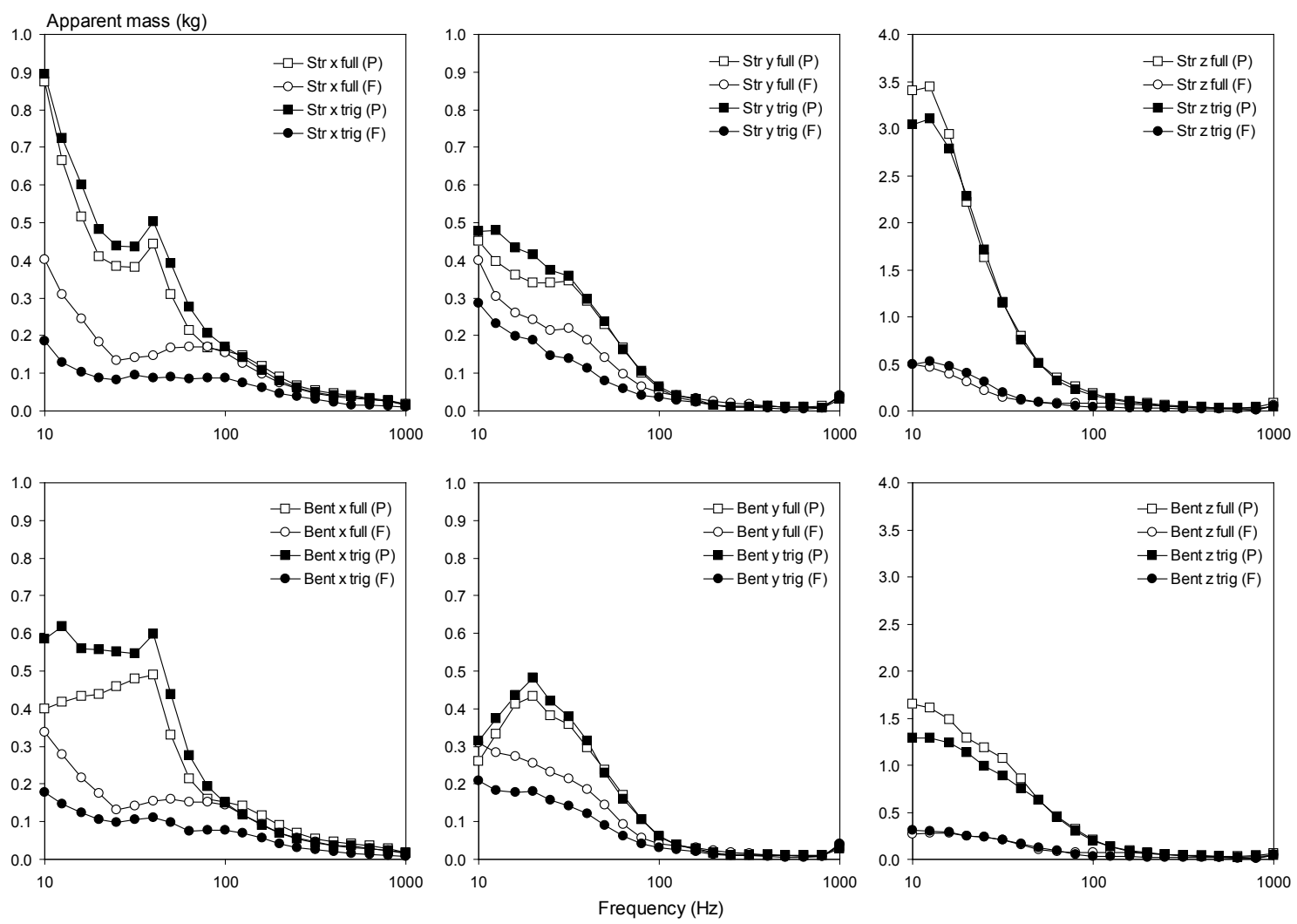

Figure 2. Mean apparent mass of the hand-arm system with straight ('str') and bent arm, with full and trigger ('trig') grip, for the finger (F) and palm (P) side of the hand. Note change of scale for $z$-axis vibration data.

as there was less finger loading. For z-axis vibration the apparent mass was about $400 \mathrm{~g}$ lower for the trigger posture (palm side) at low frequencies. For the palm side with bent arm, differences were significant at $12.5 \mathrm{~Hz}(\mathrm{p}<0.05$, Wilcoxon) but not for the straight arm. For the finger side, differences at $12.5 \mathrm{~Hz}$ were significant in the $\mathrm{x}$ - and $\mathrm{y}$-axes for both arm postures.

These results show that although slight differences occur in the apparent mass of the hand-arm system when gripping with a full and trigger posture, changes are relatively small in comparison with inter-subject variability.

\section{Acknowledgement}

This study was supported by the Great Britain Sasakawa Foundation.

\section{References}

1. Dong, R.G., Welcome, D.E., McDowell, T.W. and Wu, J.Z. (2004). Biodynamic response of human fingers in a power grip subjected to a random vibration. J. Biomechanical Engineering. $126,447-457$.

2. Shibata, N., and Maeda, S., (2009). Formulation and measurement of biodynamic forces at hand under tri-axial vibration. Proc. $43^{\text {rd }}$ UK Conference on Human Response to Vibration, Loughborough, UK. 